\title{
El plan de alta de enfermería y su impacto en la disminución de reingresos hospitalarios
}

\author{
Eloísa Tixtha López,* Adela Alba Leonel, ** Miguel Ángel Córdoba Ávila, *** Esther Mahuina Campos Castolo****
}

\section{RESUMEN}

En 1984 surgió el plan de alta en enfermería en Estados Unidos. Éste fue incluido dentro del sistema Medicare con el propósito de reducir los costos del sistema de salud, por lo que este se extendió rápidamente a Europa y América Latina. En México existen pocos estudios sobre al plan de alta en enfermería, por lo que es necesario evaluar la trascendencia y el impacto de su aplicación. Material y métodos: Se realizó un estudio transversal, descriptivo y cuantitativo en un hospital de tercer nivel. La población de estudio fueron las enfermeras con licenciatura del turno matutino. El instrumento de recolección estuvo dividido en cuatro apartados (datos generales, elaboración y contenido del plan, así como el impacto de su aplicabilidad). Resultados: Las recomendaciones del plan de alta se centran en: los medicamentos, los signos y síntomas de alarma, los cuidados en el hogar y las medidas higiénico-dietéticas con $81 \%$. Los profesionales de enfermería lo aplican de forma oral y escrita en $79 \%$. Más de 50\% no registra esta actividad en el expediente clínico. Su aplicación contribuye con el derecho que tienen los pacientes de estar informados, además del impacto en los reingresos hospitalarios. Conclusiones: La aplicación del plan de alta en enfermería es una de las formas para fomentar la reintegración del paciente a la sociedad, ya que nos proporciona una visión completa, rápida y veraz del estado de salud del paciente.

Palabras clave: Plan de alta de enfermería, aplicación, impacto, cuidados, disminución de reingresos hospitalarios, quejas y complicaciones.

\section{The nursing discharge plan and its impact on the reduction of hospital readmissions}

\begin{abstract}
Introduction: In 1984, there is the nursing discharge plan in the U.S. and this is included in the Medicare system, in order to reduce costs in the health system, so this quickly spread to Europe and Latin America. In Mexico there are few studies on the Nursing discharge plan, so it is necessary to evaluate the significance and impact of the implementation. Material and methods: We conducted a cross-sectional, descriptive, quantitative, in a tertiary hospital. The study population consisted of nurses with bachelor's morning shift. The survey instrument was divided into four sections (general information, preparation and content of the plan and the impact of its applicability. Results: The plan's recommendations focus on high:
\end{abstract}

* Licenciada en Enfermería y Obstetricia, Escuela Nacional de Enfermería y Obstetricia, UNAM.

** Maestro en Ciencias Sociomédicas con énfasis en Epidemiología. Profesor de Carrera Asociado "C", Escuela Nacional de Enfermería y Obstetricia, UNAM.

*** Gestor de Calidad. Hospital Regional de Alta Especialidad de Ixtapaluca.

***** Departamento de Investigación. Comisión Nacional de Arbitraje Médico.

Correspondencia: M en C. Adela Alba Leonel. Camino Viejo a Xochimilco y Viaducto. Tlalpan s/n, San Lorenzo Huipulco, Delegación Tlalpan, México, D.F. Tel.: 555623 32, ext. 269

E-mail: adelaalbaleonel@yahoo.com.mx

Este artículo puede ser consultado en versión completa en http://www.medigraphic.com/enfermerianeurologica 
the drugs, warning signs and symptoms, home care and lifestyle modifications with $81 \%$ nurses apply it in oral and written form by $79 \%$. More than $50 \%$ does not record this activity in the clinical record. Their application contributes to the right of patients to be informed, and the impact on hospital readmissions. Conclusions: The application of the nursing discharge plan is one way to promote the reintegration of the patient into society, as it gives us a complete, fast and accurate health status of the patient.

Key words: Nursing discharge plan, implementation, impact, care, decreased hospital readmissions, complaints and complications.

\section{INTRODUCCIÓN}

E n 1984 surgió el plan de alta en enfermería en Estados Unidos; éste fue incluido dentro del sistema Medicare con el propósito de reducir los costos del sistema de salud, por lo que éste se extendió rápidamente a Europa y América Latina ${ }^{1,2}$ por ser considerado una herramienta de gran utilidad, planificando la educación sanitaria con el único fin de incorporar al paciente en su recuperación y bienestar al egreso hospitalario.

El plan de alta en enfermería es considerado un documento de vital importancia que garantiza la continuidad de los cuidados a todos los niveles asistenciales, integrando al paciente y/o el cuidador como el eje principal del mismo. ${ }^{3}$ Según L. Carpenito, ${ }^{4,5}$ el plan del alta de enfermería es un proceso sistemático de valoración, preparación y coordinación que facilita la administración de los cuidados para la salud y asistencia social antes y después del alta del paciente. Sin embargo, para que éste logre su objetivo se requiere tanto de una acción coordinada y de colaboración entre los profesionales de la salud dentro del campo asistencial, como de la población en general.

La aplicación de este plan favorece la atención primaria, ya que permite dar continuidad a los cuidados del paciente, evita complicaciones, reingresos y quejas; ello hace que se disminuya el costo de la atención hospitalaria. El profesional de enfermería emite recomendaciones y proporciona orientación dentro del plan de alta con el fin de reincorporar al paciente a la sociedad, por lo que todo plan siempre debe brindar información de acuerdo con las necesidades del paciente en función de su diagnóstico. Asimismo, los pacientes deben recordar lo que les han enseñado y lo que han aprendido en el hospital para llevarlo a cabo en su domicilio. Debido a la gran cantidad de información que tienen que asimilar al momento del egreso es recomendable dar siempre el informe por escrito para utilizarlo como guía para el autocuidado.

El plan de alta de enfermería es considerado tan importante como el alta médica, según investigaciones realizadas en España. ${ }^{1,2}$ Este plan tiene una importante relevancia social, pues ha sido considerado como documento legal incluido en los registros clínicos de enfermería y, por ende, forma parte del expediente clínico. Cabe señalar que este plan se entrega por escrito y es totalmente independiente de la receta médica.

Derivado de su gran importancia se han realizado estudios en hospitales universitarios en los que se trata de comparar y evaluar tanto los planes antiguos de este plan como los nuevos, con el propósito de unificar criterios. En el 2003, el Hospital Universitario de Alicante, España, evaluó el diseño de nuevos formatos para documentar el plan de alta en enfermería con base en el modelo de las catorce necesidades de Virginia Henderson; este hospital encontró un aumento del porcentaje de cumplimentación de este plan de 70 a 85\%. Por su lado, el Hospital Universitario de Valencia, ${ }^{6}$ evaluó el grado de satisfacción del informe de alta para unificar los criterios e integrar la información de este plan en un solo contexto, cabe destacar que el grado de cumplimiento de este plan es de $81 \%$ en este hospital. En relación con su impacto, Alonso 7 menciona que las medidas que han demostrado evitar los reingresos hospitalarios son la aplicación del plan de alta, la educación al paciente, el seguimiento del mismo y la prevención de iatrogenias durante el ingreso previo.

Por lo anterior, una intervención del plan del alta mejora el estado de salud y permite la continuidad de los cuidados, disminuyendo así los reingresos por la misma causa en personas mayores de 65 años. Él enfatiza que la tasa de reingresos hospitalarios es más alta en los ancianos frágiles y que la insuficiencia cardiaca es uno de los problemas médicos con índice de reingresos más altos, coincidiendo con otras investigaciones, donde se establece que particularmente el anciano frágil y la insuficiencia cardiaca son uno de los problemas médicos con índices más altos de reingresos. Bajo esta premisa, Cano y colaboradores ${ }^{8}$ reportaron que el aplicar el plan de alta de enfermería en adultos mayores disminuye hasta $29.9 \%$ el número de reingresos hospitalarios, haciendo hincapié en la educación para la salud.

Lina Pinzón ${ }^{5}$ propone que la información requerida por la persona enferma debe responder adecuadamente a las necesidades generadas por su enfermedad, para que se prevengan recaídas, complicaciones o aparición de nuevas enfermedades, facilitando así, la práctica del autocuidado que derive de una vida sana y plena.

El plan de alta le permite al familiar o al cuidador conocer las recomendaciones individualizadas respecto al estado 
de salud del paciente al egreso, por lo que la comunicación enfermera-paciente y/o familia debe ser efectiva para lograr la aplicación correcta del plan de alta. Éste se debe planear desde el primer contacto con el paciente y debe concluir cuando éste sea dado de alta.

Las recomendaciones se deben proporcionar de forma oral y escrita verificando que hayan sido comprendidas en su totalidad, una vez que se haya firmado la salida por parte del médico. Cardozo ${ }^{9}$ refiere que la integración de la información al alta hospitalaria es el resultado de una buena comunicación entre los diferentes niveles de atención y propiciará un cuidado holístico a consecuencia de una comunicación efectiva del equipo multidisciplinario.

En México, existen pocos estudios sobre el plan de alta, de su aplicabilidad y cumplimentación. Con base en el diseño y validación del plan de alta en el Instituto Nacional de Cardiología "Ignacio Chávez", ${ }_{10}$ se identificaron algunas necesidades de información al egreso, tales como la dieta, el tratamiento farmacológico, entre otros con el fin de unificar los criterios en la elaboración de este plan para pacientes con afecciones cardiovasculares. Aun cuando el plan de alta en enfermería forma parte de la formación profesional de la enfermería, no existe un documento formal y estandarizado del plan de alta en enfermería para que se pueda evaluar su impacto y trascendencia. Este plan coadyuva al derecho que tienen los pacientes de estar informados tal como se menciona en la carta de los derechos generales de los pacientes ${ }^{11}$ y de acuerdo a la Ley General de Salud en su Artículo 51 Bis $1,{ }^{12}$ lo que implica, entre otros aspectos, garantizar un trato digno a los usuarios, proporcionarles información completa y una atención oportuna, evitando incurrir en implicaciones legales por omisión. Por lo anterior, es necesario evaluar la trascendencia y el impacto de la aplicación del plan de alta de enfermería, ya que éste tiene una participación crucial en la prevención de quejas, reingresos y complicaciones en los pacientes cuando egresan de la unidad hospitalaria.

\section{MATERIAL Y MÉTODOS}

Se realizó un estudio transversal en un hospital de tercer nivel. Se consideró como universo de estudio a las 138 enfermeras del turno matutino del Hospital de la Mujer; la población de estudio estuvo conformada por las licenciadas (os) en enfermería, de las(os) cuales sólo 29 cumplieron con los criterios de inclusión, a saber, tener nivel licenciatura o una especialidad con licenciatura y que estuvieran en contacto directo con el paciente. Cabe señalar que se incluyeron a los jefes de servicio, ya que éstos participan en el proceso de alta del paciente, aun cuando realizan funciones administrativas. Se tomó como criterios de exclusión a las enfermeras generales y supervisoras, ya que éstas se en- cuentran en el área administrativa y por ende no están en contacto con los pacientes. Otro de los criterios de exclusión fue los pasantes y estudiantes de enfermería.

El instrumento de recolección de información es una encuesta de opinión con 14 ítems, de los cuales 13 son de opción múltiple y una pregunta abierta. Se dividió en cuatro apartados en función de su aplicabilidad. La primera parte corresponde a datos generales como: edad, sexo, servicio y nivel de estudio. En el segundo apartado se evaluó la elaboración y aplicación del plan de alta contemplando el concepto de plan y en qué forma expresa las recomendaciones y si éstas son documentadas en el expediente clínico. La tercera parte se refiere al contenido del plan como las orientaciones acerca de los medicamentos, signos y síntomas de alarma, entre otras. Por último, se valoró la opinión del personal de enfermería sobre su impacto, las acciones que se deben implementar para incluirlo como documento legal y su pertinencia en el expediente clínico. Se obtuvo una tasa de respuesta de $100 \%$. Los reactivos se validaron mediante el consenso de expertos y la validación estadística de fiabilidad mostró una alpha de Cronbach $=0.359$, Spearman-Brown $=$ 0.069 , Guttman $=0.067$ y Lambda $=0.619$.

\section{RESULTADOS}

Nuestra población de estudio se comportó de la siguiente manera: $90 \%$ del personal de enfermería del turno matutino tienen el grado de licenciatura y $10 \%$ tiene además una especialidad; en cuanto al género, $99 \%$ corresponden al sexo femenino y el promedio de edad es de 36 años con un rango de 39 a 50 años de edad.

En lo que respecta al concepto del plan de alta, más de $50 \%$ lo define como orientación y recomendación al egreso hospitalario (Figura 1).

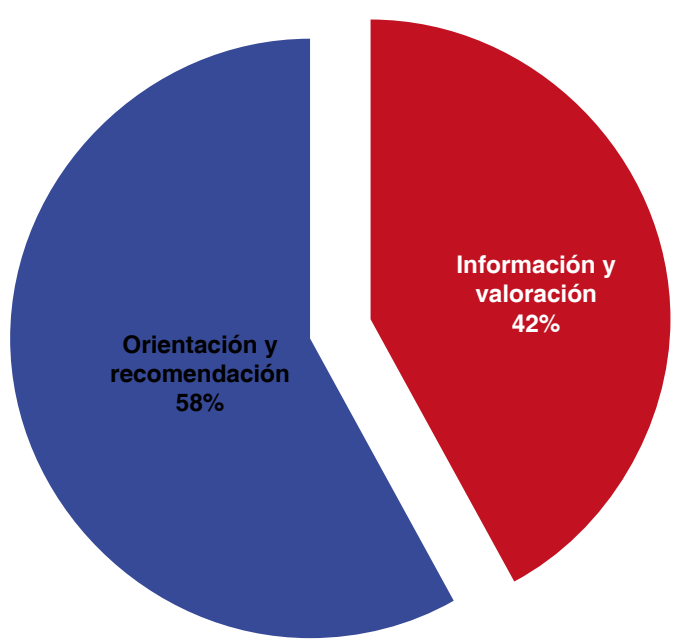

Fuente: Instrumento de Recolección de Información, 2012

Figura 1. Conceptualización del plan de alta del personal de enfermería del Hospital de la Mujer, 2012. 
Ochenta y uno por ciento de las recomendaciones están enfocadas en los medicamentos, signos y síntomas de alarma, así como los cuidados en el hogar y las medidas higiénico-dietéticas (Figura 2).

Para reforzar esta información es fundamental entregarla de forma escrita, de tal forma que el paciente pueda remitirse a ella cuantas veces sea necesario, además de usarla como guía para su autocuidado. De acuerdo con nuestros datos, la principal forma de recomendación que hace el profesional de enfermería es la expresión oral y escrita con $79 \%$ del total (Figura 3). Es importante señalar que debemos involucrar a la familia cuando se efectúan las recomendaciones al paciente, ya que al momento del egreso requiere de una fuente de apoyo para recibir la información. A pesar de ello, se tiene que

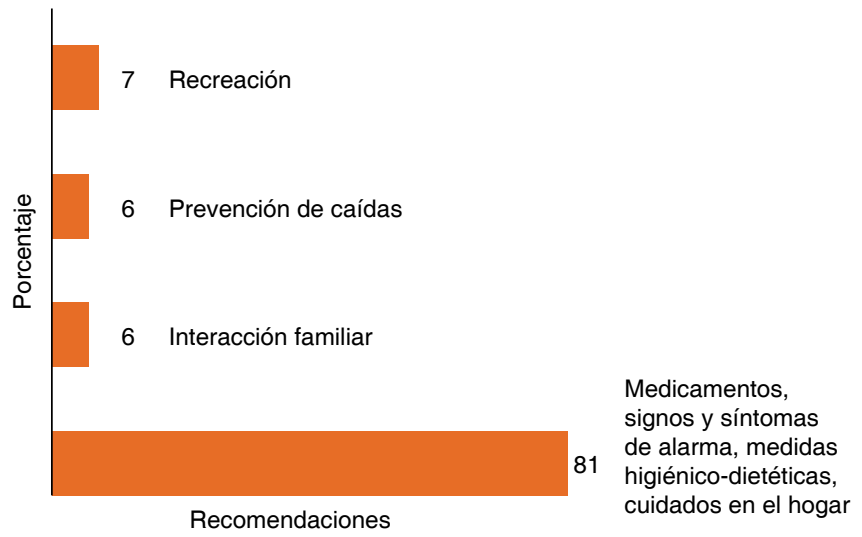

Fuente: Instrumento de Recolección de Información, 2012

Figura 2. Recomendaciones más frecuentes del personal de enfermería del Hospital de la Mujer en las pacientes al egreso, 2012.

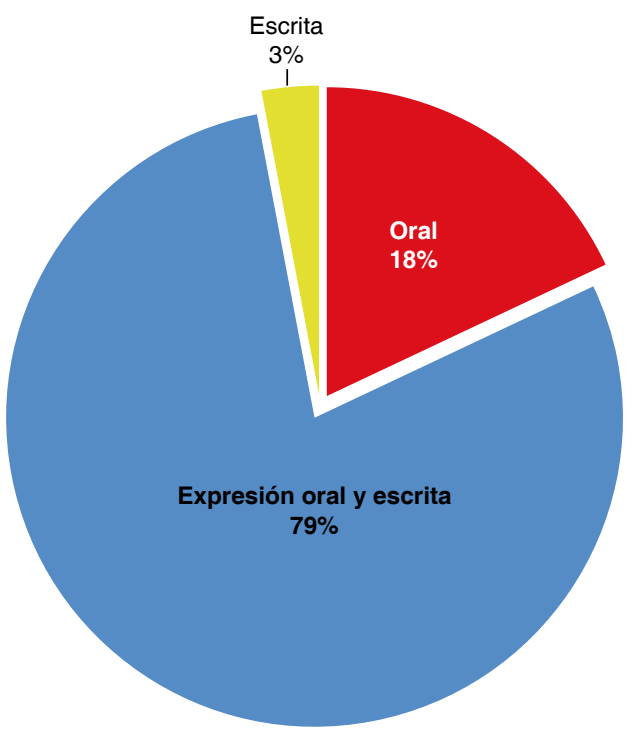

Fuente: Instrumento de Recolección de Información, 2012

Figura 3. Forma en la que realiza la recomendación el personal de enfermería del Hospital de la Mujer, 2012. tomar en cuenta que en algunos servicios, la presencia del familiar en ocasiones no es recomendable, por las condiciones de alto riesgo que se pudiesen presentar en el paciente (Figura 4).

La comunicación enfermera-paciente es fundamental, ya que permite la interacción individual. Setenta por ciento de las enfermeras aclara las dudas del paciente cuando ellas efectúan las recomendaciones al momento de su egreso y verifican que la información haya sido captada correctamente. Esto aporta nuevos conocimientos y actitudes al paciente y/o familiar sobres su estado de salud.

A pesar de ello, más de $50 \%$ de los profesionales de enfermería no documenta el plan de alta en el expediente clínico en el apartado de enfermería (Figura 5).

Cincuenta y seis por ciento del personal de enfermería sugiere que es necesario implementar el plan de alta en las instituciones como documento legal, por lo que es necesario hacer una mayor difusión de aquél y hacer más investigación al respecto. Treinta y seis por ciento considera necesario realizar guías o formatos donde se incluyan nemotecnias del plan de alta (Figura 6).

Al preguntarle al personal de enfermería cuál sería el impacto que tiene la aplicación del plan de alta, 90\% de los profesionales percibe que se disminuiría el número de quejas, reingresos hospitalarios y sobre todo de complicaciones (Figura 7).

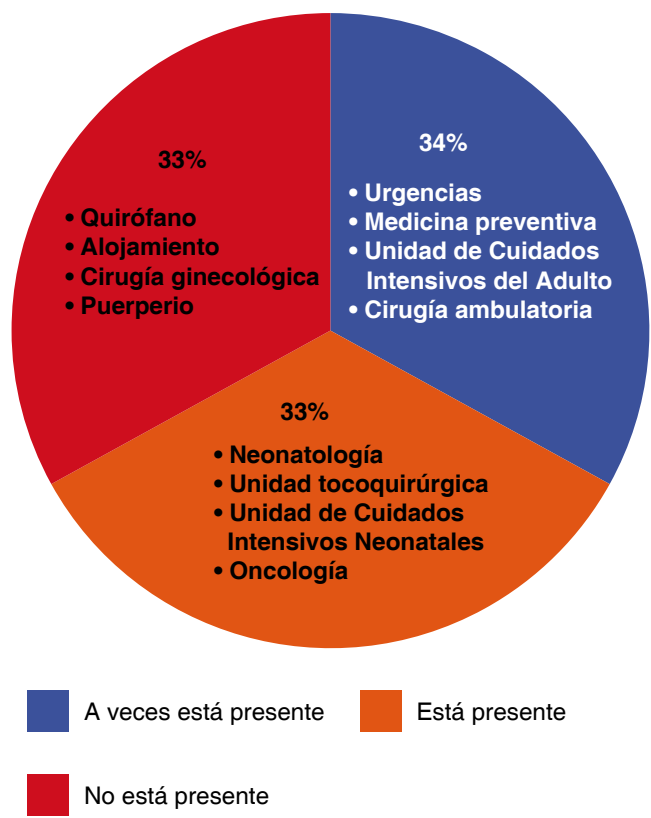

Fuente: Instrumento de Recolección de Información, 2012

Figura 4. Presencia del familiar al momento de la recomendación que da el personal de enfermería del Hospital de la Mujer, 2012. 


\section{DISCUSIÓN}

Como se demuestra en otros trabajos, más de 50\% define al plan de alta en términos generales como orientación y recomendación al egreso hospitalario; de acuerdo con L. Carpenito, ${ }^{4}$ este plan constituye un proceso sistemático de valoración, preparación y coordinación que facilita la administración de los cuidados para la salud y la asistencia social antes y después del alta. Podemos observar que existe cierta igualdad en relación con la orientación y recomendación otorgadas al egreso y que éstas se planean desde el ingreso del paciente a la unidad y finalizan cuando el mismo egresa. La información se verifica para aclarar las dudas del paciente cuando se efectúan las recomendaciones en $70 \%$;

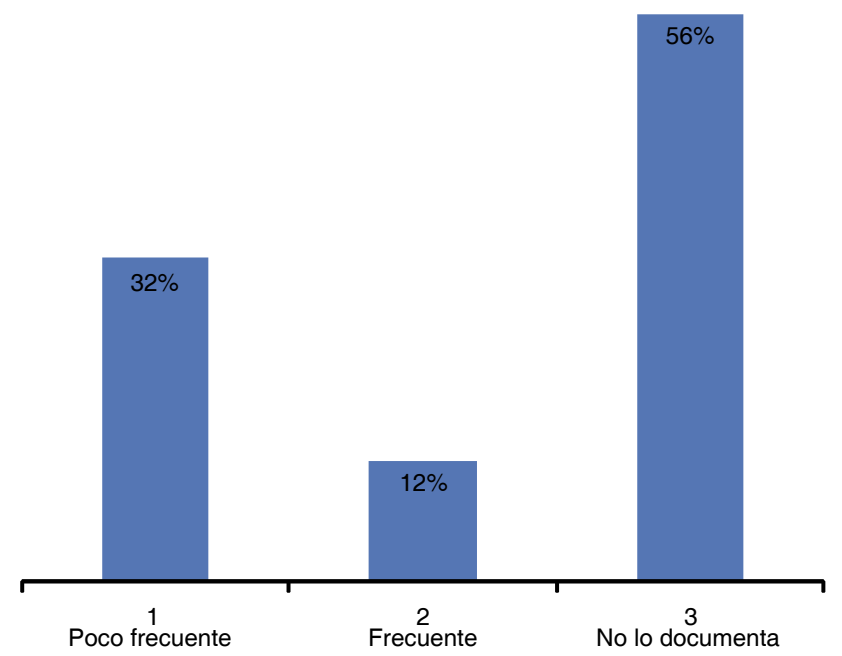

Fuente: Instrumento de Recolección de Información, 2012

Figura 5. Frecuencia de documentación del plan de alta del personal de enfermería del Hospital de la Mujer, 2012.

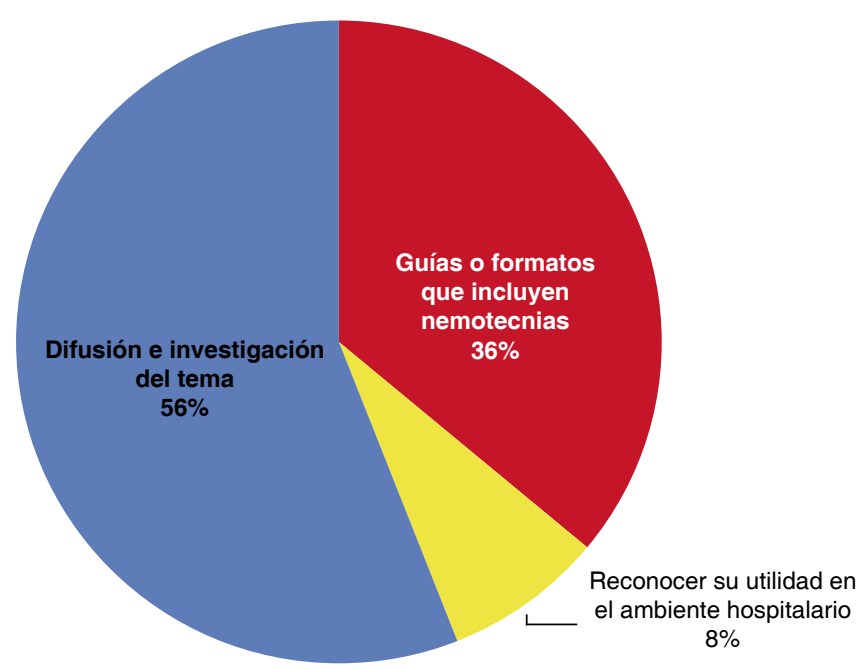

Fuente: Instrumento de Recolección de Información, 2012

Figura 6. Percepción del personal de enfermería en las acciones para implementar como documento legal el plan de alta en las instituciones, 2012. tal como lo plantea Lina Pinzón ${ }^{5}$ en función de que toda recomendación debe cumplir con ciertos criterios, tales como que sea dada de forma oral y escrita y además debe ser clara y lo más comprensible; se debe verificar que ésta haya sido entendida en su totalidad; de esta forma se garantiza su correcta aplicación por parte del paciente y/o el familiar promoviendo su autocuidado. Tal como se plantea en "El código de ética de enfermería para las y los enfermeros en México"13 en su capítulo II, artículo 7 establece que el profesional de enfermería "Fomentará una cultura de autocuidado de la salud, con un enfoque anticipatorio y de prevención del daño, propiciar un entorno seguro que prevenga riesgos y proteja a la persona".

Asimismo, con la aplicación de este plan el profesional de enfermería da un cumplimiento disciplinar a nivel internacional como se establece en Código Deontológico de Enfermería en España ${ }^{14}$ en su capítulo II, artículos 10 y 11, donde se establece que "es responsabilidad de la enfermera(o) mantener informado al enfermo, tanto en el ejercicio libre de su profesión como cuando éste se ejerce en las instituciones sanitarias, empleando un lenguaje claro y adecuado a la capacidad de comprensión del mismo", asimismo, "la enfermera/o deberá informar verazmente al paciente, dentro del límite de sus atribuciones. Cuando el contenido de esa información exceda del nivel de su competencia, se remitirá al miembro del equipo de salud más adecuado".

La presencia del familiar en el egreso del paciente tiene un papel importante, ya que éste apoya al paciente disminuyendo su estado de ansiedad, al continuar solo con sus cuidados en el hogar. Según Pérez ${ }^{15}$ el objetivo de participar en el proceso de informar es establecer una relación de confianza o una relación

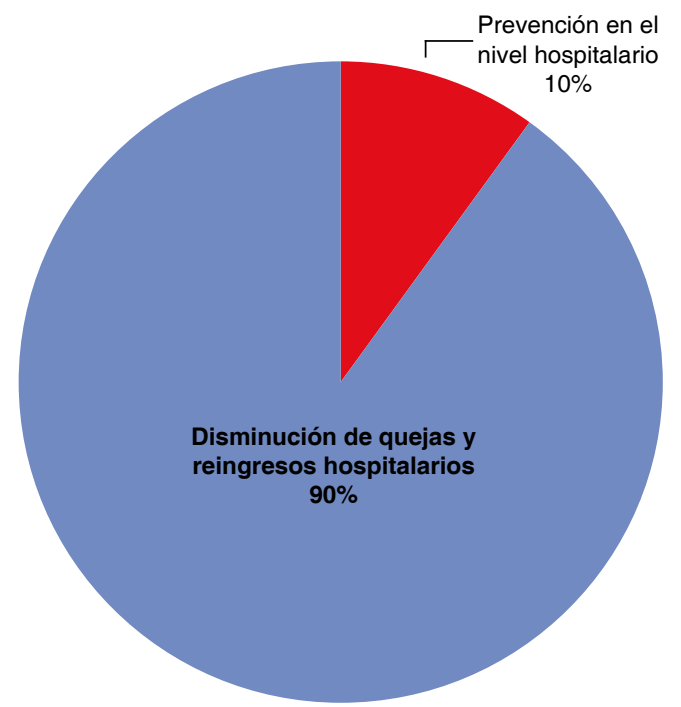

Fuente: Instrumento de Recolección de Información, 2012

Figura 7. Percepción del personal de enfermería del Hospital de la Mujer acerca del impacto de la elaboración del plan de alta, 2012. 
terapéutica para mejorar la atención a los familiares, siendo por tanto un deber de enfermería llevar a cabo este proceso. Puntunet ${ }^{10}$ identificó mediante una entrevista dirigida a los pacientes del Instituto Nacional de Cardiología algunas necesidades de información que se requieren al momento del egreso, tal como la información con respecto a la dieta con $73 \%$, tratamiento farmacológico $70 \%$ y problema de salud actual con 60\%; de acuerdo con esta información el paciente puede continuar con su rehabilitación en el hogar. Estas recomendaciones son muy similares a nuestro estudio, ya que $81 \%$ están enfocadas a los medicamentos, signos y síntomas de alarma, cuidados en el hogar y medidas higiénico-dietéticas.

El registro de enfermería constituye la principal fuente de información, ya que nos brinda una visión completa y global del cuidado del paciente, para la continuidad de los cuidados, evitando duplicidad de los mismos, mejorando así la comunicación. En nuestro estudio $70 \%$ realizó las recomendaciones de forma oral y escrita, sin embargo, $50 \%$ no lo documentó en el expediente clínico. Aun cuando $90 \%$ del personal encuestado es de nivel de licenciatura y $10 \%$ con especialidad.

El plan de alta en España es considerado como un documento legal, sin embargo, se realizó un estudio de investigación con el propósito de conocer si éste se registraba dentro del expediente clínico y, de acuerdo con los datos de Talamante ${ }^{6}$ se menciona que sólo $81 \%$ del personal de enfermería sí lo registra en el expediente clínico y éste lo utilizan para dar continuidad a los cuidados del paciente y mejorar la comunicación entre los profesiones de salud.

Asimismo en México, todo registro debe ser legible, evitando el uso de abreviaturas y términos que no sean reconocidos, tal como se establece en la NOM-004SSA3-2012 del Expediente Clínico (antes denominada NOM-168-SSA1-1998). El registro se debe realizar adecuadamente, para demostrar la legalidad de los actos realizados: legalmente lo que no se escribe no se hizo. ${ }^{16}$ De este modo se evita que el personal de enfermería caiga en desviaciones que incurren en una falta penal como la omisión, tal como se establece en la reglamentación del artículo $5^{\circ}$ Constitucional de la Ley General de Profesiones, en su artículo 71 establece que "los profesionales serán civilmente responsables de las controversias que cometan en el desempeño de su trabajo". Así como en el artículo 33, "está obligado a poner sus conocimientos científicos y recursos al servicio de su cliente". ${ }^{17}$

Para implementar el plan de alta en las instituciones como documento legal en nuestro país, el personal de enfermería del Hospital de la Mujer considera que es necesario hacer una mayor difusión de éste, así como hacer más investigación al respecto y aplicarlo de acuerdo con los diferentes niveles de atención. Su aplicación según Puntunet ${ }^{10}$ implica un cambio en el actuar del profesional de enfermería, pues cambia la concepción que la persona tiene sobre la profesión al descubrir que ella brinda cuidados de forma integral y autónoma.

Noventa por ciento de los enfermeros en nuestro estudio, perciben que el plan de alta tendría un impacto en la disminución de quejas y reingresos hospitalarios, tal como lo plantea Cano, ${ }^{8}$ donde evidencian que, mediante la educación para la salud y el seguimiento vía telefónica, disminuye en $29.9 \%$ los reingresos tras la implementación planificada del alta en pacientes mayores de 65 años. De igual forma Alonzo $^{7}$ ha demostrado que la aplicación del plan de alta entre otras medidas disminuye el número de reingresos, por lo que, una intervención individualizada dentro del plan del alta, mejora el estado de salud y permite la continuidad de los cuidados, disminuyendo así los reingresos por la misma causa en personas mayores de 65 años.

Si se disminuyen los reingresos, se aumenta la calidad de vida, garantizando así su pronta recuperación y reintegración a la sociedad y, por ende, se disminuyen el número de quejas, por lo que el plan de alta lo debemos considerar como una herramienta fundamental para brindar cuidados de enfermería. Para lograr esto, es necesario que todo el personal de enfermería aplique el plan de alta.

\section{CONCLUSIONES}

La aplicación del plan de alta en enfermería es una de las formas para fomentar el autocuidado y la reintegración del paciente a la sociedad, ya que nos proporciona una visión completa, rápida y veraz del estado de salud del paciente, así como de los cuidados enfermeros que requiere.

Por lo que es necesario que tanto las escuelas y facultades de enfermería incluyan al plan de alta de enfermería dentro de los programas de estudio, ya que éste es una herramienta fundamental para dar continuidad a los cuidados de enfermería y así, evitar en la medida de lo posible los reingresos hospitalarios, disminución de quejas y complicaciones. Es por esto que este plan se debe proporcionar de forma oral y escrita al paciente y/o a su familia cuando egrese y éste debe ser lo más claro $\mathrm{y}$ entendible posible.

\section{AGRADECIMIENTOS}

A la jefatura de Investigación del Hospital de la Mujer por el apoyo en la recolección de información. Asimismo a todo el personal de enfermería de esa unidad hospitalaria, ya que sin su colaboración no hubiera sido posible realizar esta investigación. 


\section{BIBLIOGRAFÍA}

1. Santamaría-Fernández MB, Duque-López EA. Eficacia de la planificación del alta de enfermería en pacientes mayores de 65 años con patología cardíaca [internet]. Madrid, España: Fundación Hospital Alcorcon; 2007 [Acceso 20-01-2013]. Disponible en: http: //www.fac.org.ar/qcvc/llave/ c112e/santamariam.php

2. Navarro-Arnedo JM, Orgiler-Uranga PE, De Haro-Marín S. Nuevo informe de alta de enfermería de cuidados intensivos: grado de satisfacción y cumplimentación de los colectivos implicados. Rev Enferm Colín. 2006; 16(4): 190-197.

3. Ube-Pérez JM, Urzanqui-Rodríguez M, Concepción-Rodríguez $\mathrm{H}$, Blasco-Ríos M, Ascarza-Rubio S, Musitu-Pérez V. Evaluación de la relevancia del informe de enfermería al alta hospitalaria por el usuario interno y externo. Rev Nure Investigación. 2006; (24): 1-23.

4. Carpenito-Lynda J. Diagnósticos de Enfermería. 3a ed. Madrid, España: Interamericana Mc-Graw Hill; 2000: 170-172.

5. Pinzón-Salazar L. El plan de alta: una herramienta para el cuidado integral y la recuperación de la persona enferma. [Acceso 21-02-2013]. Disponible en: http//promocionsalud.ucaldas.edu.co/dolados/revis.php

6. Talamante-Sierra C, Sayas-Chulia V, Famoso-Poveda MJ, MuñozIzquierdo A, Peiro- Andres A et al. Continuidad de cuidados: Alta en enfermería. Rev Enferm Cardiol. 2007; 41: 35-40.

7. Alonso-Martínez JL, Llorente-Diez B, Echegaray-Agara M, UrbietaEchezarreta MA, González-Arencibia C. Reingreso Hospitalario en Medicina Interna. Rev An Med. Interna. 2001; (18): 28-34.

8. Cano-Arana A, Martín-Arribas MC, Martínez-Piedrola M, García-Talles $\mathrm{C}$, Hernández-Pascual $\mathrm{M}$ et al. Eficacia de la planificación del alta de enfermería para disminuir los reingresos en mayores de 65 años. Atención primaria[Inertnet]. [Acceso 26-03- 2013]. Disponible en: http: //www. sciencedirect.com/science?_ob=ArticleURL\&_udi=B94RY
9. Cardozo-Gonzales RI, Scatena-Villa TC, Nunes-Alves L, Laecher-Caliri MH, Midori-Sassaki C. Gerenciamiento de caso de enfermería en el proceso de alta hospitalaria del paciente con lesión medular. Rev Mex Enfer Cardiol. 2000; 8: 7-13.

10. Puntunet BM, López FL, Domínguez BA, Cadena EJC, Ruiz AC, Teniza ND. Diseño y validación de un formato general para documentar el plan de alta en la persona con alteración. Rev Mex Enfer Cardiol. 2010; (18): 19-24.

11. Carta de los derechos generales de las pacientes y los pacientes 2001 [internet]. [Acceso 30-01-2013] Disponible en https://www.conamed. gob.mx/publicaciones/pdf/derechos_pacientes.pdf

12. Secretaria de Salud. Ley General de Salud en México [internet]. México: Secretaría de salud; 2006 [Acceso 10 enero 2013] Disponible en https:// www.salud.gob.mx/unidades/cdi/legis/lgs/index-indice.htm

13. Secretaría de Salud. Código de Ética para las Enfermeras y Enfermeros en México [Internet]. México: SSA; 2001. [Acceso 20-02- 2013]. Disponible en: https://www.ssa.gob.mx/código/enfermería. México_pdf

14. Organización Colegial de Enfermería. Código Deontológico de la Enfermería Española [Internet]. España: Universidad de Navarra; 2003 [Acceso 25-01-2013]. Disponible en: https://www.unav.es/cdb/esotcodigoenf.html

15. Pérez-Fernández MC, Navarro-Infante FR, Dulce-García MA, GallardoJiménez N, Fernández-Fernández A. Comunicación: una necesidad para el paciente-familia. Una competencia de Enfermería. Revista Páginasenferurg.com. 2009; (13): 15-20.

16. Norma Oficial Mexicana NOM-004-SSA3-Del Expediente Clínico. México: Diario Oficial de la Federación 2012. [Acceso 02-03-2013]. Disponible en: https://www.conamed.gob.mx/publicaciones/pdf/norma_oficial.pdf.

17. Cordova-Ávila MA. Legislación en los servicios de enfermería [Internet]. México: SSA; 2009. [Acceso 22-02-2013]. Disponible en https://www. smme.org.mx/documentacion/LegislacionEnfermeria.pdf 